\title{
Routine practice in the diagnosis of adenocarcinoma of the stomach: a survey of tumours diagnosed in the Portsmouth and Oxford Health Districts 1979-1980
}

\author{
M. Deakin, ${ }^{1}$ D.G. Colin-Jones ${ }^{1}$ and M.P. Vessey ${ }^{2}$ \\ ${ }^{1}$ Department of Gastroenterology, Queen Alexandra Hospital, Cosham, Portsmouth and ${ }^{2}$ Department of \\ Community Medicine and General Practice, Radcliffe Infirmary, Oxford, UK.
}

\begin{abstract}
Summary: If the poor prognosis for patients with adenocarcinoma of the stomach is to be improved, it is important to make the diagnosis at an early stage. In order to evaluate our routine diagnostic practice, we have performed a retrospective study of patients in the Portsmouth and Oxford Health Districts who were diagnosed as having adenocarcinoma of the stomach during 1979 and 1980.

Three hundred and forty-two patients were identified; the great majority had advanced disease at the first presentation. Only 9 patients $(2.6 \%)$ were identified as having early gastric carcinoma and a total of 46 patients $(13.4 \%)$ had either Stage 1 or 2 disease. Despite the provision of an endoscopy service, gastric carcinoma is still being diagnosed at an advanced stage.

Initial false negative investigations caused a delay in diagnosis in $18.4 \%$ of patients. During the year preceding diagnosis (when tumour was likely to have been present), the false negative rate for barium meal examination was $25.5 \%$ and for endoscopy $13.5 \%$.

Negative results should not deter us from pursuing the diagnosis of tumour in those patients with persisting symptoms.
\end{abstract}

\section{Introduction}

In the early 1970s, Japanese workers reported encouraging survival rates for patients with carcinoma of the stomach, particularly those with early gastric cancer. ${ }^{1}$ As a result, it was hoped that the growing use of endoscopy in Britain would improve accuracy in diagnosing carcinoma of the stomach and would increase the detection of early gastric cancer, leading to better survival rates.

Clinical reviews of carcinoma of the stomach conducted in the era before the establishment of endoscopic services, ${ }^{2,3}$ showed that most tumours were advanced at diagnosis and that early gastric cancer made up less than $1 \%$ of tumours diagnosed. Since early gastric cancer can remain confined to the mucosa for as long as 5 years $^{4}$ and the prognosis of advanced tumours is poor, ${ }^{5,6}$ it is important that gastric carcinoma is diagnosed at the first presentation.

The purpose of this study was to examine the mode of presentation of patients, using a detailed

Correspondence: D.G. Colin-Jones, M.D., F.R.C.P. Accepted: 12 August 1987 retrospective assessment, and to attempt to evaluate modern diagnostic methods. Data have been collected about patients diagnosed as suffering from adeno-carcinoma of the stomach in the years 1979 and 1980 in two health districts, Portsmouth and Oxford, where both endoscopy and radiology were available.

\section{Patients and methods}

The notes of all patients diagnosed as having gastric adenocarcinoma in 1979 and 1980 in the Portsmouth Health District, and all except those treated at the Horton Hospital in Banbury in the Oxford Health District, were examined retrospectively. Records were identified from the following sources: (1) hospital activity analysis; (2) gastroscopy clinic records; (3) cancer registry; (4) pathology department records; (5) registered deaths; (6) our cimetidine post-marketing surveillance study. ${ }^{7}$ Data for each patient were transferred onto a standard form to allow further analysis.

(C) The Fellowship of Postgraduate Medicine, 1988 


\section{Staging}

Early gastric cancer was defined as carcinoma of the stomach in which invasion was limited to the mucosa, or to the mucosa and sub-mucosa, irrespective of lymph node involvement. ${ }^{8}$ In Stage 1 , the tumour did not extend into the muscularis propria; in Stage 2, the muscularis propria was involved but lymph nodes did not contain tumour; in Stage 3, lymph nodes contained tumour irrespective of the depth of invasion of the primary. Stage 4 tumours either had proven distant spread or were invading surrounding structures. ${ }^{9}$

\section{Results}

The estimated populations of the two districts during the study period were 510,000 in Portsmouth and 501,000 in Oxford. During the two years 1979-1980, 342 patients were diagnosed as having carcinoma of the stomach, 198 in Portsmouth and 144 in Oxford. Male patients predominated $2: 1$ and the disease tended to be diagnosed at a younger age in males than females.

\section{Stage distribution}

Nine $(2.6 \%)$ of the patients were shown to have early gastric cancer (8 Stage 1 , one Stage 3 ). Eight patients $(2.3 \%)$ had Stage 1 disease, 38 patients $(11.1 \%)$ had Stage 2 disease, 53 patients $(15.5 \%)$ had Stage 3 disease and 142 patients $(41.6 \%)$ had Stage 4 disease. Staging was not possible with accuracy in 101 further patients $(29.5 \%)$ as neither a laparotomy nor a post-mortem examination was performed. This group of patients, however, had advanced gastric tumours clinically; $83 \%$ died within 6 months of diagnosis, and none survived longer than 18 months. The two year survival rates for the patients studied with staged tumours were $100 \%$ for Stage 1, 58\% for Stage 2, $26 \%$ for Stage 3 and $5 \%$ for Stage 4 . The 2 year survival rate for the 342 patients was $14.3 \%$. All patients with early gastric cancer were alive at 2 years without signs of recurrence.

\section{Presentation}

Most patients presented with 'classical' symptoms; the earliest symptoms were most commonly weight loss and abdominal pain, as found by others. ${ }^{10}$ In $72 \%$, symptoms had been present for less than 3 months before seeing their general practitioners. Referral from general practice was usually prompt but $16 \%$ of patients were followed for longer than 3 months. When patients were referred for hospital consultation, $60 \%$ of patients were seen within 1 week and $91 \%$ within 6 weeks of receipt of a referral letter.

\section{Previous history of upper gastrointestinal disease}

Seven patients had been known previously to have had a gastric ulcer (median length of history 7 years; range 1-21 years). Five patients had had previous gastric and duodenal ulcers $(28 ; 9-38$ years) and 23 patients had a history of duodenal ulceration (23; 2-55 years). In a further 12 patients there was a history of ulceration but the site had not been accurately documented $(20 ; 1-49$ years). Seventeen patients $(5 \%)$ had previously undergone gastric surgery ( $33 ; 4-55$ years).

Thirteen patients $(4 \%)$ were known to have a family history of carcinoma of the stomach; this included 4 of the 9 patients with early tumours.

\section{Diagnosis}

The carcinoma was first diagnosed by barium meal in 180 patients, by endoscopy in 116 patients, by laparotomy in 24 patients, by biopsy of distant metastases in 6 patients and at post-mortem examination in 16 patients.

In assessing the diagnostic methods we have assumed that cancer was, in fact, present for at least a year before diagnosis.

\section{Initial investigation by barium meal}

During the year up to diagnosis 232 patients underwent 255 barium meals. A firm diagnosis of cancer was made in 171 cases, there were 10 patients in whom the barium meal was doubtful (including 1 patient who had an earlier negative barium meal) and there were 65 barium meals in 52 patients where tumour was not suspected; a false negative rate of $25.5 \%$ for cancer detection.

In the 52 patients where the diagnosis of cancer was not established by the first barium meal, no pathology was detected in 21 , a hiatus hernia in 6 , gastric ulceration believed to be benign in 16 , duodenal pathology in 6 , and miscellaneous conditions in 3. Endoscopy was subsequently performed in 36 of these 52 patients and the diagnosis of cancer was made in 35 although more than one endoscopy was required to establish the diagnosis in five patients. Sixteen patients had no endoscopic follow up and were eventually diagnosed by repeat barium meal in 9 , by surgery in 5 and at post-mortem in 2 with a median delay of 18 weeks (range, 1 to 50 weeks). 


\section{Initial investigation by endoscopy}

Upper gastrointestinal endoscopy was performed in 125 patients and 141 occasions in the year before diagnosis. A diagnosis sufficiently certain to prompt action was made in 108 cases at the first examination when both macroscopic appearance and histology of multiple biopsies had been taken into account. One further patient had a doubtful endoscopy which was repeated obtaining positive histology. In 19 endoscopies in 16 patients the diagnosis of carcinoma was missed (false negative rate $13.5 \%$ ). No pathology was found in 1 patient, 9 patients had lesser curve gastric ulcers, 1 patient had a pre-pyloric ulcer, 1 had pyloric stenosis, and 4 had strictures at the gastro-oesophageal junction believed to be benign. Non-malignant histology was reported in the 11 of these 16 patients in whom biopsies had been taken. Diagnosis of tumour was eventually made in 9 patients at follow-up endoscopy, in 2 by barium meal and in 5 at surgery.

In 17 cases macroscopically benign lesions were found to be malignant on histology and in 19 cases doubtful or negative histology was obtained in patients with obvious macroscopic tumours. A definitive tissue diagnosis was achieved in $83 \%$ of cases.

Five patients had negative endoscopy and negative barium meals in the year before diagnosis. Three had benign gastric ulcers which were observed and biopsied but in two a separate mucosal lesion was subsequently noted and biopsies showed malignancy - one was an early gastric cancer.

In the year before diagnosis, therefore, 63 patients had been investigated without detection of a possible malignancy. Some patients had had earlier barium meals or endoscopies. The relationship of these negative investigations to the five year period before diagnosis is shown in Table I.

\section{Discussion}

Adenocarcinoma of the stomach remains a disease of the late middle-aged and elderly, the age and sex

Table I Previous negative investigations

\begin{tabular}{lcccccccc}
\hline & \multicolumn{3}{c}{$\begin{array}{c}\text { Months prior to } \\
\text { diagnosis }\end{array}$} & \multicolumn{5}{c}{$\begin{array}{c}\text { Years prior to } \\
\text { diagnosis }\end{array}$} \\
& $<3$ & $3-6$ & $7-12$ & $1-2$ & $2-3$ & $3-4$ & $4-5$ \\
\hline $\begin{array}{c}\text { Barium meals } \\
\text { performed }\end{array}$ & 45 & 4 & 16 & 16 & 7 & 6 & 4 \\
$\begin{array}{c}\text { Endoscopies } \\
\text { performed }\end{array}$ & 12 & 2 & 5 & 2 & 1 & 5 & 2 \\
\hline
\end{tabular}

distributions we have observed being similar to those reported in earlier series. ${ }^{11}$ Presenting symptoms are well known (notably weight loss and abdominal pain) and tend to occur late in the disease. In this series, $72 \%$ of patients presented to their general practitioner with symptoms of less than 3 months duration. This figure may be biased through patients minimizing the length of their history and is higher than the $49 \%$ of patients presenting within 3 months described by Brookes $e t$ al. in a similar retrospective study. ${ }^{6}$ Contrary to previous reports ${ }^{2}$ we found that the small number of patients with early tumours also presented with typical histories.

Although a little better than before the availability of endoscopy, ${ }^{2}$ the proportion of early gastric carcinomas $(2.6 \%)$ was lower than had been hoped for, and Stage 1 and 2 tumours were found in only $13.3 \%$ of the patients. Only 49 patients survived for 2 years after diagnosis and 20 of these are known to have died subsequently. This is a disappointing result and we can only conclude that our current practice has had little influence on the outcome of carcinoma of the stomach. Most gastric cancers appear to cause symptoms late in their growth but major delays in diagnosis may also occur which are in part attributable to the patients (20\% presented to their general practitioners more than 3 months after symptoms started), in part to late referral from general practice $(16 \%$ of patients first saw their doctors for 3 months or longer before referral), and in part to falsely negative investigations (in $18.4 \%$ of the patients).

\section{How then can we improve our practice?}

\section{Screening asymptomatic patients}

Screening for gastric cancer is practised in some countries, such as Japan, with apparent success, but its value has not been proven by a randomized controlled trial.

In Great Britain, mass screening, even if effective, would be difficult to justify on economic grounds except perhaps in high risk areas such as Wales or in high risk patient groups. ${ }^{12}$ A small genetic influence on the risk of carcinoma of the stomach has been reported which appears to be accentuated by smoking. ${ }^{13}$ Patients with a family history may develop the tumour at an earlier age than others. ${ }^{14}$ In this study 4 of the 9 patients with early tumours had a family history of carcinoma of the stomach; they may have presented early because of increased awareness of the significance of symptoms. 


\section{Early investigation of symptomatic patients}

In this study patients mainly at risk were males of age greater than 45 years and females of age greater than 55 years.

Education to reduce delay in diagnosis might in the first place alert patients above these ages to the importance of weight-loss and new dyspeptic symptoms. Clearer warnings might, for example, be provided with proprietary antacid preparations and pharmacists might alert patients to this problem. Secondly it is necessary for doctors to recognise (a) the importance of early upper gastrointestinal investigation in older patients with minimal dyspeptic symptoms, weight loss, anaemia or vague abdominal pains, (b) the need to avoid the prescription of ulcer healing drugs without a definitive diagnosis in the older patient; treatment of patients with $\mathrm{H}_{2}$ receptor antagonists can provide symptomatic relief ${ }^{15}$ and this can only lead to delay in diagnosis, (c) the value of endoscopy if there is clinical suspicion of carcinoma of the stomach even if the barium meal is negative or shows an apparently benign lesion. ${ }^{16}$

\section{Reduction of hospital waiting lists}

We did not identify a major problem in this study of patients waiting for clinic appointments. Long waiting lists for hospital appointments might have been expected to have been a factor in delaying the diagnosis. In practice it seems that the patients were usually referred for urgent consultation or at times directly admitted.

\section{Improvement in the accuracy of diagnostic methods}

Despite the acceptance and throughput of the endoscopy units in both Portsmouth and Oxford, it is clear that during the years 1979 and 1980, barium meals were being used as the major screening investigation for the elderly with suspected tumours especially in Oxford; 255 barium meals were performed within 1 year of diagnosis with a false negative rate of $25.5 \%$ for the identification of tumours. Although higher than reported in special prospective studies of barium meals ${ }^{17}$ this rate is comparable with other studies of barium meals in routine use. ${ }^{18-20} \mathrm{~A}$ high false

\section{References}

1. Kidokoro, T. Frequency of resection, metastasis and 5 year survival of early gastric cancer in a surgical clinic. In: Murakami, T. (ed) Early Gastric Cancer. University of Tokyo Press, Tokyo, 1972, pp 55-59. negative rate inevitably leads to delay until the diagnosis is reconsidered.

There were 19 instances where endoscopy was falsely negative for tumour in the year prior to diagnosis $(13.5 \%)$. On 18 occasions, however, the actual lesion was seen but wrongly thought to be benign, whereas with barium studies, the lesion was missed or a coincidental lesion was commonly reported to account for symptoms.

The problem of benign versus malignant gastric ulcer was a feature in 16 patients who were believed to have benign disease on barium meal. This problem is widely recognized and despite careful evaluation of barium meals, ${ }^{21}$ endoscopic biopsy in addition is indicated in patients with gastric ulcers ${ }^{16}$ - a view supported by our experience. Biopsy of some lesions in the stomach, however, particularly lesser curve gastric ulcers and strictures at the gastro-oesophageal junction, can be difficult and technique still needs to be improved. ${ }^{22-24}$

In conclusion, we continue to diagnose most cases of gastric carcinoma at a stage when treatment can only be palliative. Both endoscopy and radiology gave falsely negative results in routine clinical practice in two large health districts. Any delay in establishing the diagnosis must be minimized if more Stage 1 or 2 lesions are to be found. The false negative results contributed to delays in diagnosis in no less than $18.4 \%$ of the cases investigated but the errors arose in different ways - radiologists missed lesions completely whilst endoscopists observed them only to conclude erroneously that they were benign. Development of double contrast techniques and endoscopic biopsy methods may help (especially with gastric ulcers) but awareness of the failings of the two diagnostic modalities also seems important if more cases of gastric cancer are to be diagnosed at a treatable stage.

\section{Acknowledgements}

We thank Mrs Shirley Wood and Mr T. Lucas for all their help in conducting this study and the consultants in the Portsmouth and Oxford Health Districts who have allowed us to present these data concerning patients under their care. We are grateful to Smith Kline and French Laboratories Ltd for their financial support with this project.

2. Fielding, J.W., Ellis, D.J., Jones, B.G. et al. Natural history of early gastric cancer: results of a 10 year regional survey. Br Med J 1980, 281: 965-967. 
3. Evans, D.M.D., Craven, J., Murphey, F. \& Cleary, B.K. Comparison of 'early gastric cancer' in Britain and Japan. Gut 1980, 19: 1-9.

4. Tsukuma, H., Mishima, T. \& Oshima, A. Prospective study of 'early' gastric cancer. Int J Cancer 1983, 31: 421-426.

5. Larsen, M. Gastric carcinoma. Am Surg 1983, 49: 105-109.

6. Brookes, V.S., Waterhouse, J.A.H. \& Powell, D.J. Carcinoma of the stomach: a 10 year survey of results and factors affecting prognosis. $\mathrm{Br}$ Med J 1965, 00: 1577-1573.

7. Colin-Jones, D.G., Langman, M.J.S., Lawson, D.H. \& Vessey, M.P. Cimetidine and gastric cancer: preliminary report from post marketing surveillance study. Br Med J 1982, 285: 1311-1313.

8. Tasaka, S. Statistical study of early gastric cancer, collected throughout Japan. Japanese Society of Gastroenterology. Gastroenterol. Endoscopy (Tokyo) 1962, 4: 1-16.

9. Fielding, J.W.L. et al. Clinicopathological staging of gastric cancer. Br J Surg 1984, 71: 677-680.

10. Houghton, P.W.J. et al. Early gastric cancer: the case for long term surveillance. $\mathrm{Br} \mathrm{Med} J$ 1985, 291: 305308.

11. Swynnerton, B.F. \& Truelove, S.C. Carcinoma of the stomach. Br Med J 1952, 1: 287-292.

12. Logan, R.F.A. \& Langman, M.J.S. Screening for gastric cancer after gastric surgery. Lancet 1983, ii: 667-670.

13. Hoshino, H., Hirayama, T., Arimoto, et al. Gastric cancer risk factors: a case controlled study based on medical records. Jpn $J$ Cancer Res 1985, 76: 846-850.

14. Lynch, H.T., Kruch, A.J., Thomas, R.J. \& Lynch, J. Cancer family syndrome. In: Lynch, H.T. (ed) Cancer Genetics. Charles C. Thomas, Illinois, 1976, pp 355388.
15. Elder, J.B., Ganguli, P.C. \& Gillespie, I.E. Cimetidine and gastric cancer. Lancet 1979, i: 1005-1006.

16. Mountford, R.A., Brown, P., Salmon, P.R. et al. Gastric cancer detection in gastric ulcer disease. Gut 1980, 21: 9-17.

17. Keto, P., Suoronto, H., Ihamaha, T. \& Melartin, E. Double contrast examination of the stomach compared with endoscopy. Acta Radiol [Diagn] (Stockh) 1979, 20: 762-768.

18. Cotton, P. Fibreoptic endoscopy and the barium meal: results and implications. $B r \operatorname{Med} J$ 1973, 2: 161165.

19. Sekons, D.H., McSherry, C.K., Calthorn, W.F., Pudalov, B., Beatow, H.L. \& Hirami Shinya. Contribution of endoscopy to diagnosis and treatment of gastric cancer. Am J Surg 1984, 147: 662-665.

20. Dooley, C.P., Larson, A.W., Stace, N.H. et al. Double-contrast barium meal and upper gastrointestinal endoscopy. A comparative study. Ann Int Med 1984, 101: 538-545.

21. Salter, R.H., Gill, D.K., Girdwood, T.G., McNeil, R.H. \& Athey, G. Gastric ulcers, is endoscopy always necessary? Br Med J 1981, 282: 2097.

22. Dekker, W. \& Tytgat, G.N. Diagnostic accuracy of fiber-endoscopy in the detection of upper intestinal malignancy: a follow up analysis. Gastroenterology 1977, 73: 710-714.

23. Graham, D.Y., Swartz, J.T., Cain, G.D. \& Gyorkey, F. Prospective evaluation of biopsy number in the diagnosis of esophageal and gastric cancer. Gastroenterology 1982, 82: 228-231.

24. Danesh, B.J.Z., Burke, M., Newman, J., Aylott, A., Whitfield, P. \& Cotton, P.B. Comparison of weight, depth, and diagnostic adequacy of specimens obtained with 16 different biopsy forceps designed for upper gastrointestinal endoscopy. Gut 1985, 26: 227-231. 